\title{
A Robust Fractal Color Image Watermarking Algorithm
}

\author{
Jian Lu, Yuru Zou, Chaoying Yang, and Lijing Wang \\ College of Mathematics and Computational Science, Shenzhen University, Shenzhen 518060, China \\ Correspondence should be addressed to Yuru Zou; yrzou@163.com
}

Received 23 September 2013; Accepted 16 December 2013; Published 2 March 2014

Academic Editor: Yuncai Wang

Copyright (C) 2014 Jian Lu et al. This is an open access article distributed under the Creative Commons Attribution License, which permits unrestricted use, distribution, and reproduction in any medium, provided the original work is properly cited.

\begin{abstract}
One of the main objectives of watermarking is to achieve a better tradeoff between robustness and high visual quality of a host image. In recent years, there has been a significant development in gray-level image watermarking using fractal-based method. This paper presents a human visual system (HVS) based fractal watermarking method for color images. In the proposed method, a color pixel is considered as a 3-D vector in $R G B$ space. And a general form of $3 \times 3$ matrix is utilized as the scaling operator. Meanwhile, the luminance offset vector is substituted by the range block mean vector. Then an orthogonalization fractal color coding method is achieved to obtain very high image quality. We also show that the orthogonalization fractal color decoding is a mean vectorinvariant iteration. So, the range block mean vector is a good place for hiding watermark. Furthermore, for consistency with the characteristics of the HVS, we carry out the embedding process in the CIE $L a^{*} b^{*}$ space and incorporate a just noticeable difference (JND) profile to ensure the watermark invisibility. Experimental results show that the proposed method has good robustness against various typical attacks, at the same time, with an imperceptible change in image quality.
\end{abstract}

\section{Introduction}

With the rapid development of internet, digital multimedia data such as image, audio, and video are readily reproduced and distributed with ease. Many watermarking methods have been exploited for copyright protection due to the properties of digital media and the popularity of the internet. Typically, a watermark can be a random signal, a meaningful message, or a logo. It is viewed as an effective way to prevent user's content from illegal distribution [1]. Figure 1 shows a general scheme for digital watermarking. Firstly, an encrypted watermark is inserted into an original image by using an embedding algorithm. Then, the watermarked image will pass through the transmission channel, which may include some attacks, such as lossy compression, low-pass filtering, noising, and geometric distortions. When the watermark is to be extracted, the corresponding extraction algorithm will be implemented, usually with aid of a secret key and the original image. Only the owner of the image knows the key and no other person can identify the watermark without the knowledge of the key. A good watermarking scheme should possess many important properties, for example, invisibility, robustness to various types of attacks, and accurate detection.
In general, digital image watermarking algorithms addressing a wide variety of applications can be classified into two categories depending on the domain in which the watermark is embedded. The first group is mainly implemented in spatial domain $[3,4]$ while the second group of algorithms is achieved in transform domain, such as discrete cosine transform (DCT) $[5,6]$, discrete Fourier transform (DFT) [7] and discrete wavelet transform (DWT) $[8,9]$. The watermark in spatial domain methods is inserted into the image by directly modifying a preselected set of pixels in the image. On the other hand, in transform domain watermarking methods, the watermark is embedded into the image by modifying the transformed frequency coefficients. The advantage of watermarking in the frequency domain is that the characteristics of the human visual system (HVS) are better captured by the spectral coefficients, since the HVS is more sensitive to low-frequency coefficients and less sensitive to high-frequency coefficients $[10,11]$. When focusing on color image watermarking, many methods are realized by embedding watermarks in the image luminance or by processing each color channel separately. By observing the fact that human eyes are insensitive to changes in the blue component, Kutter et al. [12] proposed a method for embedding a 


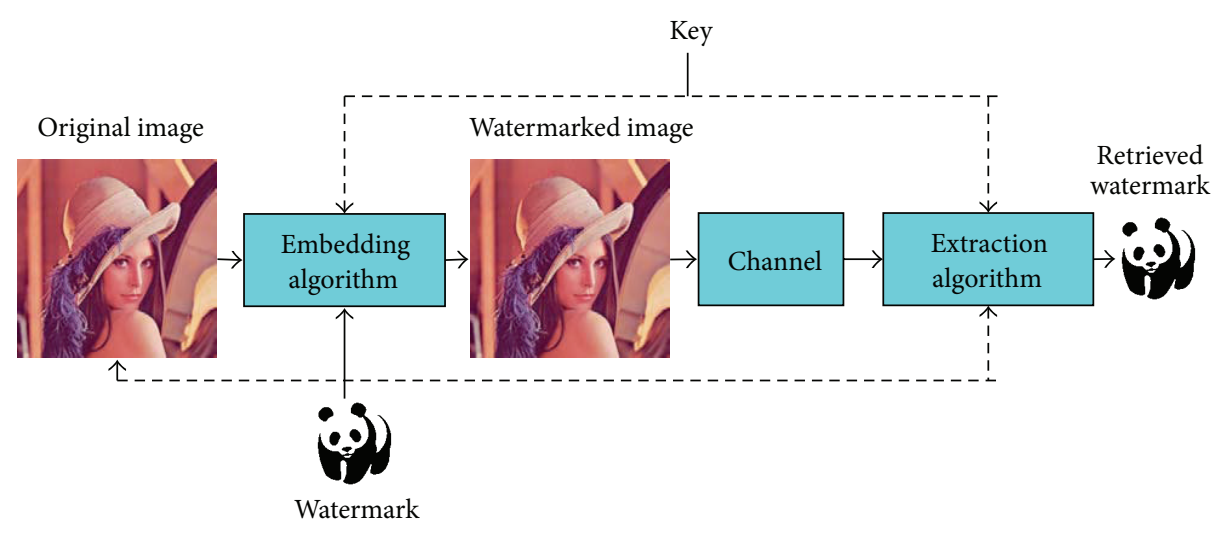

FIGURE 1: Generic watermark insertion and extraction procedure.

watermark in the blue component. Barni et al. [13] introduced a color image watermarking scheme based on the cross-correlation of $R G B$ channels. In Barni's scheme, a watermark is embedded within the host image by modifying a subset of full frame DCT coefficients of each color channel. And the existence of the watermark is verified by calculating the correlation of $R G B$ channels. Tsai et al. [14] provided a scheme of embedding a watermark on a quantized color image. Tsui et al. [15] introduced a solution of embedding a watermark in the frequency domain of the chromatic channels by using the spatiochromatic discrete fourier transform (SCDFT). In Tsui's method, the chromatic content of a color image is encoded into CIE $a^{*} b^{*}$ chromaticity coordinates while the achromatic content is encoded as CIE $L$ tristimulus value. Kuo and Cheng [16] presented a color watermarking method that combines color edge detection and color quantization using principal axes analysis in 3-D color space. Chou and Liu [17] introduced a color image watermarking scheme that hides watermark signals in most distortion-tolerable signals within three color channels of the host image without resulting in perceivable distortion in wavelet domain. Also, Vahedi et al. [18] proposed a watermarking scheme of color images based on wavelet transformation. In Vahedi's method, the strength of the embedded watermark is controlled locally with the aid of the visual characteristics of the host image and so forth.

The idea of fractal image coding (FIC) was originally introduced by Barnsley and Demko [19]. And in 1992, Jacquin [20] achieved the first practical fractal image coding method. In the past decades, fractal image coding has mainly been developed for the purpose of image compression [21-33] as well as some for image denoising [34-37] and image indexing [38]. Recently, it is also well studied for gray-level image watermarking [39-43]. Davern and Scott [39] described a steganographic method for inserting secret information into images using fractal image coding. The method allowed a user to specify a visual key when hiding the secret information. The visual key must then be used when retrieving the hidden data. Candik et al. [40] investigated two possible approaches of embedding digital watermarks into fractal codes of images, which are embedding digital watermarks into parameters for position of similar blocks and coefficients of block similarity.
Kiani and Moghaddam [41] presented a watermarking method to embed a binary watermark into an image using a special type of fractal coding that its parameters are contrast scaling and the mean of range block. And it utilized the fuzzy $C$-mean clustering to address the watermark bits. Pi et al. [42] studied a novel watermarking method utilizing a special type of orthogonalization fractal coding. In the method, a permutated pseudorandom binary sequence used as watermark was embedded into the range block means. And the detecting procedure was carried out by computing the correlation coefficient between the original and the extracted watermark. Shahraeini and Yaghoobi [43] proposed a blind watermarking algorithm based on fractal model in discrete wavelet domain and so forth.

However, few known papers considered fractal coding for watermarking of color images. In this paper, motivated by [42], we advance a HVS-based fractal color image watermarking algorithm. Meanwhile, an analogous $R G B$ version of orthogonalization fractal coding is introduced. Just like other watermarking methods, good robustness and high image quality are our goals. In our proposed method, based on the observation that the $r, g$, and $b$ components for a natural image are correlated, we consider a pixel as a $3-D$ vector in $R G B$ space. Then, instead of using three independent linear predictions for the pixel intensity of the three planes (namely, $r, g$, and $b$ planes) in the classic fractal color coding [2], we adopt a general form by using a $3 \times 3$ matrix as the scaling factor. In addition, the range block mean vector substitutes the luminance offset vector. In terms of the quality of the fractal representation measured by PSNR, the proposed fractal coding significantly performs better than the traditional fractal color image coding. We also show that orthogonalization fractal color decoding is a mean-invariant iteration. So, we use the range block mean vectors to hide the watermark. On the other hand, in order to accord with the characteristics of the HVS, we convert the range mean vectors from $R G B$ space to CIE $L a^{*} b^{*}$ space before the inserting process (with the aid of the JND model [44]) is carried out. The experimental results show that the proposed method is very robust against different attack, as well as with retaining very high image quality. 


\section{Foundations of Fractal Image Coding}

The theory of fractal-based image coding using iterated function system (IFS) and collage theorem was proposed by Barnsley and Demko [19]. Let $(X, d)$ be a complete metric space. An iterated function system (IFS) is a finite set of contraction mappings $w_{i}: X \rightarrow X$, where $i=1,2, \ldots, N$ with contraction factor $s_{i}$ on $(X, d)$. In general, the system is denoted by $\left\{X ; w_{1}, w_{2}, \ldots, w_{N}\right\}$ and the contraction factor is $s=\max \left\{s_{1}, s_{2}, \ldots, s_{N}\right\}$. Furthermore, we define $W: \mathscr{H}(X)$ $\rightarrow \mathscr{H}(X)$ as $W(B)=\bigcup_{i=1}^{N} w_{i}(B), \forall B \in \mathscr{H}(X)$. Then, the transformation $W$ is a contraction mapping on the complete metric space $(\mathscr{H}(X), h)$, where $h$ is the Hausdorff metric. So, according to the contractive mapping fixed-point theorem, there exists a unique fixed point $A \in \mathscr{H}(X)$ satisfying

$$
\widetilde{A}=W(\widetilde{A})=\bigcup_{i=1}^{N} w_{i}(\widetilde{A})
$$

which is known as an attractor of the IFS. The fixed point can be obtained by following iteration

$$
\widetilde{A}=\lim _{n \rightarrow \infty} W^{(n)}(B), \quad \forall B \in \mathscr{H}(X),
$$

where $W^{(n)}(B)=W\left(W^{(n-1)}(B)\right)$ and $W^{(0)}(B)=B$. Let $\epsilon \geq 0$ be a small given real number. With the above hypothesis, collage theorem says that, suppose $L \in \mathscr{H}(X)$ satisfying $h\left(L, \bigcup_{i=1}^{N} w_{i}(L)\right) \leq \epsilon$; then,

$$
h(L, \widetilde{A}) \leq \frac{\epsilon}{1-s} .
$$

The collage theorem implies that if a set can approximate to contracted copies of itself as well as possible by a set of contractive mappings, then the corresponding attractor is close enough to the set. Thus, the IFS code for the set can be stored since the approximator can be obtained by iterating the IFS on any initial set.

However, in practice it is unlikely to find "collages" for most natural images by reasonably simple transforms. The well-known practical method for fractal image coding, namely partitioned iterated function system (PIFS), was proposed by Jacquin [20]. Let $I$ be a given gray-level image, and hence $I$ is a subset in three-dimensional Euclidian space that is, $I \in \mathbb{R}^{3}$. The image $I$ is first segmented into $n$ nonoverlapping range blocks of size, say $b \times b$, and denoted by $\mathscr{R}=$ $\left\{R_{i}\right\}_{i=1}^{n}$. Note that $I=\bigcup_{i=1}^{n} R_{i}$ and $R_{i} \cap R_{j}=\varnothing$ for any $i \neq j$. Let $\mathscr{D}=\left\{D_{j}\right\}_{j=1}^{m}$ be the pool of all domain blocks which are of size $2 b \times 2 b$ and all are extracted from $I$ that is, $D_{j} \subset I$ for $j=$ $1, \ldots, m$. Let $\Phi_{j}=\left\{\phi: \phi\left(D_{j}\right) \rightarrow \mathbb{R}^{3}\right\}$, where $\phi$ is usually an affine contractive transformation. For each range block $R_{i}$, let $d$ be distance measure. Our goal is to find a domain block $D_{k}$ as well as an appropriate transformation $\phi_{i \mid k}$ such that

$$
d\left(R_{i}, \phi_{i \mid k}\left(D_{k}\right)\right)=\min _{j}\left\{d\left(R_{i}, \phi_{i \mid j}\left(D_{j}\right)\right)\right\},
$$

where $\phi_{i \mid j} \in \Phi_{j}$ and $\phi_{i \mid j}$ satisfies

$$
d\left(R_{i}, \phi_{i \mid j}\left(D_{j}\right)\right) \leq d\left(R_{i}, \phi\left(D_{j}\right)\right), \quad \forall \phi \in \Phi_{j} .
$$

When all the range blocks are processed, the set of maps $\Phi=$ $\left\{\phi_{i \mid}\right\}_{i=1}^{n}$ thus is obtained and called the PIFS code of the image I.

In classic fractal coding, the distance $d$ always uses $l^{2}$ norm, $\|\cdot\|_{2}$, as the so-called collage distance measure. The affine contractive map $\phi$ consists of three parts defined as

$$
\phi=\varphi \circ \tau \circ \omega .
$$

Here, $\omega$ is a uniform scaling from domain block to range block dimension; $\tau$ is one of the eight isometries that map a block into itself (identity, $90^{\circ}, 180^{\circ}$, and $270^{\circ}$ rotations around the center and the four reflections over the symmetry axes); and $\varphi$ is a linear transform in the gray level, defined as

$$
\varphi(x)=\alpha x+\beta,
$$

where $\alpha$ and $\beta$ are known as the contrast and luminance offset parameters, respectively, and $\alpha$ is usually restricted to satisfy $|\alpha|<1$ for contractivity. (Some authors, e.g., [21], have restricted $|\alpha|<\sqrt{2}$ with no noticeable effects on the contractivity.)

\section{Fractal Color Image Coding}

The traditional fractal color image coding method in $R G B$ space discussed in [2] for the purpose of compression was performed by using three gray-level linear predictions to encode the $r, g$, and $b$ image components independently. It was defined as

$$
\varphi(\mathbf{x})=\operatorname{diag}\left(\left[\begin{array}{lll}
\alpha_{1} & \alpha_{2} & \alpha_{3}
\end{array}\right]\right) \mathbf{x}+\mathbf{b},
$$

where $\mathbf{x}=\left[\begin{array}{lll}x_{1} & x_{2} & x_{3}\end{array}\right]^{T}$ represents $(r, g, b)$ values of a pixel and $\mathbf{b}=\left[\begin{array}{lll}b_{1} & b_{2} & b_{3}\end{array}\right]^{T}$ denotes the corresponding offset values. However, for most natural images, the components $r, g$ and $b$ are always strongly correlate. For instance, when testing Lena image, the correlations between $r$ and $g, r$ and $b, g$ and $b$ are $0.8786,0.6764$ and 0.9106 , respectively. The correlation of $r, g$ and $b$ is 0.9881 , which is estimated by [45]

$$
\rho=\sqrt{1-\frac{|\Sigma|}{\prod_{i}\left|\Sigma_{i i}\right|}}
$$

where $\Sigma$ is the covariance matrix of vectors $r, g$ and $b$. Likewise for Barbara image, the correlations between $r$ and $g, r$ and $b, g$ and $b$ are $0.8860,0.8061$ and 0.9582 , respectively; the correlation of $r, g$ and $b$ is 0.9921 . Based on this observation, in this work, with above notations, we consider the $r, g, b$ components as a whole and adopt a more general functional form:

$$
\varphi(\mathbf{x})=\mathbf{A x}+\mathbf{b},
$$

where the linear operator $\mathbf{A}$ is defined by a $3 \times 3$ matrix:

$$
\mathbf{A}=\left[\begin{array}{lll}
a_{11} & a_{12} & a_{13} \\
a_{21} & a_{22} & a_{23} \\
a_{31} & a_{32} & a_{33}
\end{array}\right] .
$$


In general, the distance between the pixels $\mathbf{x}$ and $\mathbf{y}$ is defined by using $l^{2}$-norm (Euclidean distance), that is,

$$
d_{2}(\mathbf{x}, \mathbf{y})=\|\mathbf{x}-\mathbf{y}\|_{2}=\sqrt{\sum_{i=1}^{3}\left(x_{i}-y_{i}\right)^{2}}
$$

Let $\mathbf{x}_{k} \doteq\left[\begin{array}{lll}x_{1 k} & x_{2 k} & x_{3 k}\end{array}\right]^{T}$ and $\mathbf{y}_{k} \doteq\left[\begin{array}{lll}y_{1 k} & y_{2 k} & y_{3 k}\end{array}\right]^{T}, k=1, \ldots$, $K$, where $K=\# R_{i}$ denoting the pixel number of $R_{i}$, be the $(r$, $g$, and $b$ ) values on $R_{i}$ and transformed $D_{j}$, respectively. Then the distance of $\left\{\mathbf{x}_{k}\right\}$ and $\left\{\mathbf{y}_{k}\right\}$ can be determined by

$$
\begin{aligned}
\Delta_{i, j}^{2} & =\sum_{k=1}^{K} d_{2}^{2}\left(A \mathbf{x}_{k}+\mathbf{b}, \mathbf{y}_{k}\right) \\
& =\sum_{k=1}^{K}\left\|A \mathbf{x}_{k}+\mathbf{b}-\mathbf{y}_{k}\right\|_{2}^{2} \\
& =\sum_{k=1}^{K} \sum_{u=1}^{3}\left(\sum_{v=1}^{3} a_{u, v} x_{v, k}+b_{u}-y_{u, k}\right)^{2} .
\end{aligned}
$$

The purpose of using squared $l^{2}$ norm in function (13) is to facilitate the estimation of the matrix A. According to the matrix theory, formula (13) can be represented using the well known (squared) Frobenius norm; that is,

$$
\Delta_{i, j}^{2}=\|\mathbf{A X}+\mathbf{b I}-\mathbf{Y}\|_{F}^{2}
$$

where $\mathbf{b I} \doteq\left[\begin{array}{llll}b_{1} \mathbf{I} & b_{2} \mathbf{I} & b_{3} & \mathbf{I}\end{array}\right]^{T}$ and $\mathbf{I}=\left[\begin{array}{llll}1 & 1 & \cdots & 1\end{array}\right]^{T}$ being a $K \times 1$ vector. $\mathbf{X} \doteq\left[\begin{array}{lll}X_{1} & X_{2} & X_{3}\end{array}\right]^{T}, X_{v}=\left[\begin{array}{llll}x_{v 1} & x_{v 2} & \cdots & x_{v K}\end{array}\right]^{T}$, and $v=$ $1,2,3$ denote the respective $r, g, b$ values of the pixels in the domain block $D_{j}$. Likewise, $\mathbf{Y} \doteq\left[\begin{array}{lll}Y_{1} & Y_{2} & Y_{3}\end{array}\right]^{T}, Y_{v}=$ $\left[\begin{array}{llll}y_{v 1} & y_{v 2} & \cdots & y_{v K}\end{array}\right]^{T}$, and $v=1,2,3$ denote the respective $r, g$, and $b$ values of the pixels of $R_{i}$.

In order to minimize the residual $\Delta_{i, j}^{2}$, we rewrite (13) as

$$
\Delta_{i, j}^{2}=\sum_{u=1}^{3}\left\|\sum_{v=1}^{3} a_{u, v} X_{v}^{T}+b_{u} \mathbf{I}-Y_{u}^{T}\right\|_{2}^{2}
$$

and differentiate it with respect to $b_{u}, a_{u, v}$ and set them to zero; that is, $\partial \Delta_{i, j}^{2} / \partial b_{u}=0$ and $\partial \Delta_{i, j}^{2} / \partial a_{u, v}=0$ yielding

$$
\begin{aligned}
& b_{u}= \frac{1}{K} \sum_{k=1}^{K} y_{u, k}-a_{u, 1} \frac{1}{K} \sum_{k=1}^{K} x_{1, k} \\
&-a_{u, 2} \frac{1}{K} \sum_{k=1}^{K} x_{2, k}-a_{u, 3} \frac{1}{K} \sum_{k=1}^{K} x_{3, k} \\
& \doteq \bar{Y}_{u}-a_{u, 1} \bar{X}_{1}-a_{u, 2} \bar{X}_{2}-a_{u, 3} \bar{X}_{3} \quad u=1,2,3, \\
& a_{u, 1} \sum_{k=1}^{K} x_{1, k} x_{v, k}+a_{u, 2} \sum_{k=1}^{K} x_{2, k} x_{v, k} \\
&+a_{u, 3} \sum_{k=1}^{K} x_{3, k} x_{v, k}+b_{u} \sum_{k=1}^{K} x_{v, k}=\sum_{k=1}^{K} y_{u, k} x_{v, k},
\end{aligned}
$$

that is,

$$
\begin{array}{r}
a_{u, 1} X_{1}^{T} X_{v}+a_{u, 2} X_{2}^{T} X_{v}+a_{u, 3} X_{3}^{T} X_{v}+b_{u} K \bar{X}_{v}=Y_{u}^{T} X_{v}, \\
u, v=1,2,3,
\end{array}
$$

where $\bar{Y}_{u}=(1 / K) \sum_{k=1}^{K} y_{u, k}$ and $\bar{X}_{v}=(1 / K) \sum_{k=1}^{K} X_{u, k}, v=$ $1,2,3$. Inserting (16) into (18), we have

$$
\begin{aligned}
& {\left[\begin{array}{lll}
a_{u, 1} & a_{u, 2} & a_{u, 3}
\end{array}\right] \cdot\left[\begin{array}{c}
X_{1}^{T} X_{v}-K \bar{X}_{1} \bar{X}_{v} \\
X_{2}^{T} X_{v}-K \bar{X}_{2} \bar{X}_{v} \\
X_{3}^{T} X_{v}-K \bar{X}_{3} \bar{X}_{v}
\end{array}\right]=Y_{u}^{T} X_{v}-K \bar{Y}_{u} \bar{X}_{v}} \\
& u, v=1,2,3 .
\end{aligned}
$$

For a test image, let the $r, g, b$ values $X_{u}=\left\{x_{u, k}\right\}_{k=1}^{K}$ and $Y_{u}=\left\{y_{u, k}\right\}_{k=1}^{K}, u=1,2,3$ be random samples of the random variables $X_{u}$ and $Y_{u}$ representing the pixel value distribution of the parent subblock and the child subblock, respectively. Then, the formula (19) can be comprehensively written as follows:

$$
\mathrm{AC}=\mathrm{M}
$$

where $\mathbf{C}$ is a covariance matrix

$$
\begin{gathered}
\mathbf{C}=\left[\begin{array}{ccc}
D\left(X_{1}\right) & \operatorname{Cov}\left(X_{1}, X_{2}\right) & \operatorname{Cov}\left(X_{1}, X_{3}\right) \\
\operatorname{Cov}\left(X_{1}, X_{2}\right) & D\left(X_{2}\right) & \operatorname{Cov}\left(X_{2}, X_{3}\right) \\
\operatorname{Cov}\left(X_{1}, X_{3}\right) & \operatorname{Cov}\left(X_{2}, X_{3}\right) & D\left(X_{3}\right)
\end{array}\right], \\
\mathbf{M}=\left[\begin{array}{lll}
\operatorname{Cov}\left(Y_{1}, X_{1}\right) & \operatorname{Cov}\left(Y_{1}, X_{2}\right) & \operatorname{Cov}\left(Y_{1}, X_{3}\right) \\
\operatorname{Cov}\left(Y_{2}, X_{1}\right) & \operatorname{Cov}\left(Y_{2}, X_{2}\right) & \operatorname{Cov}\left(Y_{2}, X_{3}\right) \\
\operatorname{Cov}\left(Y_{3}, X_{1}\right) & \operatorname{Cov}\left(Y_{3}, X_{2}\right) & \operatorname{Cov}\left(Y_{3}, X_{3}\right)
\end{array}\right] .
\end{gathered}
$$

As a result, if $\mathbf{C}$ is an invertible matrix, which can be easily satisfied by randomly adding extremely small positive real numbers to each element of $\mathbf{C}$, then

$$
\mathbf{A}=\mathbf{M} \cdot \mathbf{C}^{-1} \text {. }
$$

Moreover, summarizing (16) gives

$$
\mathbf{b}=\overline{\mathbf{Y}}-\mathbf{A} \overline{\mathbf{X}},
$$

where $\overline{\mathbf{Y}}=\left[\overline{Y_{1}} \overline{Y_{2}} \overline{Y_{3}}\right]^{T}$ and $\overline{\mathbf{X}}=\left[\overline{X_{1}} \overline{X_{2}} \overline{X_{3}}\right]^{T}$.

According to the Banach's fixed point theorem, in order to guarantee the contractivity of the fractal transformation with respect to $l^{2}$ norm, $\mathbf{A}$ should satisfy the restriction $\|\mathbf{A}\|_{2}<1$, which implies that the maximum eigenvalue $\lambda$ of $\mathbf{A}^{T} \mathbf{A}$ must be smaller than one. There is no simple relationship between the coefficients of $\mathbf{A}$ and $\lambda$. However, in the $l^{\infty}$ norm, contractivity is guaranteed if all A satisfy the condition $\sum_{v=1}^{3}\left|a_{u, v}\right|<1$ for each $u \in\{1,2,3\}$. The resulting fractal transforms satisfying this condition are always contractive in $l^{\infty}$, hence in $l^{2}$, due to the equivalence of the norms in finite pixel space. For this reason, we will "clamp" the coefficients of A by checking each row of $\mathbf{A}$ if it satisfies $\sum_{v=1}^{3}\left|a_{u, v}\right|<1$. If $\sum_{v=1}^{3}\left|a_{u, v}\right| \geq$ 1 for some $u \in\{1,2,3\}$, we re-encoded the $u$-component $Y_{u}$ of 
the child subblock $R_{i}$ by "discarding" one or two unimportant coefficient(s) in $\left\{a_{u, v}\right\}_{v=1}^{3}$. Firstly, the correlation coefficients between $Y_{u}$ and $X_{1}, X_{2}, X_{3}$ of the corresponding parent subblock $D_{j}$ are calculated and denoted by $\rho_{1}, \rho_{2}, \rho_{3}$, respectively. Secondly, the magnitudes of the three correlations are sorted in ascending order, for example, $\left|\rho_{v_{1}}\right| \geq\left|\rho_{v_{2}}\right| \geq\left|\rho_{v_{3}}\right|$, which means that $X_{v_{1}}$ has maximum correlativity. Finally, we "clamp" the coefficients $a_{u, v}, v=1,2,3$ by following way:

(i) If $\rho_{v_{2}}$ has the same sign as $\rho_{v_{1}}$, then set $a_{u, v_{3}}=0$ and

$$
\begin{aligned}
& {\left[\begin{array}{l}
a_{u, v_{1}} \\
a_{u, v_{2}}
\end{array}\right]^{T}} \\
& \quad=\left[\begin{array}{l}
\operatorname{Cov}\left(Y_{u}, X_{v_{1}}\right) \\
\operatorname{Cov}\left(Y_{u}, X_{v_{2}}\right)
\end{array}\right]^{T}\left[\begin{array}{cc}
D\left(X_{v_{1}}\right) & \operatorname{Cov}\left(X_{v_{1}}, X_{v_{2}}\right) \\
\operatorname{Cov}\left(X_{v_{1}}, X_{v_{2}}\right) & D\left(X_{v_{2}}\right)
\end{array}\right]^{-1} .
\end{aligned}
$$

If $\left|a_{v_{1}}\right|+\left|a_{v_{2}}\right| \geq 1$, goto (iv).

(ii) If $\rho_{v_{2}}$ and $\rho_{v_{1}}$ have different sign whereas $\rho_{v_{3}}$ and $\rho_{v_{1}}$ take the same sign, then set $a_{u, v_{2}}=0$ and $a_{v_{1}}, a_{v_{3}}$ are computed with the similar equation as (24) by replacing $X_{v_{2}}$ with $X_{v_{3}}$. If $\left|a_{v_{1}}\right|+\left|a_{v_{3}}\right| \geq 1$, goto (iv).

(iii) If both $\rho_{v_{2}}$ and $\rho_{v_{3}}$ have different sign with $\rho_{v_{1}}$, then goto (iv).

(iv) Set $a_{u, v_{2}}=a_{u, v_{3}}=0$ and $a_{u, v_{1}}=\operatorname{Cov}\left(Y_{u}, X_{v_{1}}\right) / D\left(X_{v_{1}}\right)$. If $\left|a_{u, v_{1}}\right|>1$, then $a_{u, v_{1}}$ is "clamped" to $a_{u, v_{1}}=$ $\operatorname{sign}\left(a_{u, v_{1}}\right) * 0.99$.

When the "clamped" A is determined, b can be calculated naturally by (23). Accordingly, the (nonorthogonalization) fractal decoding algorithm with $\mathbf{A}$ and $\mathbf{b}$ is given by

$$
R^{(n)}=\mathbf{A} \omega\left(D^{(n-1)}\right)+\mathbf{b} \mathbf{I} .
$$

If $\mathbf{A}$ and $\bar{R}$ are taken as fractal parameters, the orthogonalization decoding algorithm is

$$
\begin{aligned}
R^{(n)} & =\mathbf{A} \omega\left(D^{(n-1)}\right)+\mathbf{b} \mathbf{I} \\
& =\bar{R} \mathbf{I}+\mathbf{A} \omega\left(D^{(n-1)}-\overline{D^{(n-1)}} \mathbf{I}\right),
\end{aligned}
$$

where $\bar{R}$ denotes the mean of $r, g$, and $b$ components of the range block $R$; that is, $\bar{R} \doteq\left[\begin{array}{lll}R_{1} & \bar{R}_{2} & \bar{R}_{3}\end{array}\right]^{T} ; D^{(n-1)}$ is the matched domain block from the $(n-1)$ th iteration and $\overline{D^{(n-1)}} \doteq \overline{D_{1}^{(n-1)}} \overline{D_{2}^{(n-1)}}{\overline{D_{3}^{(n-1)}}}^{T}$. From (26), it can be readily verified that $R^{(n)}$ is a mean vector-invariant iteration; that is,

$$
\overline{R^{(n)}}=\overline{R^{(n-1)}}=\cdots=\bar{R} \text {. }
$$

In Figure 2, we test the proposed method on the $512 \times 512$ color Lena (Figure 2(a)), Barbara (Figure 2(d)), and Peppers (Figure 2(g)) images using full search scheme. We set the size of range blocks to be $4 \times 4$ and the search step size to be 2 . We can see that, in terms of PSNR qualitative measures, the image quality in Figures 2(b), 2(e), and 2(h) with PSNRs $34.71 \mathrm{~dB}$, $30.37 \mathrm{~dB}$, and $32.01 \mathrm{~dB}$ encoded by the method proposed here is significantly better than the quality of Figures 2(c), 2(f), and 2(i) encoded by the traditional fractal image coding in [2], around $2.0 \mathrm{~dB}$.

\section{Fractal Color Watermarking}

In general, there are two types of techniques for embedding a predefined watermark into an image. One is pseudorandom sequence used for objective detection; the other is binary image or gray image used for subjective detection [1]. In this paper, in order to subjectively verify the ownership of an image with the aid of extracting a watermark, a binary logo image is used. And the watermark bit is either 1 or 0 . One of the main challenges of the watermarking is to achieve a tradeoff between robustness and perceptivity. In general, increasing the strength of the embedded watermark can achieve robustness, but it would lead to an increase in the visible distortion as well, and vice versa. Since the orthogonalized fractal decoding is a mean-invariant iteration, the range block mean is a good robust place to hide a watermark, as discussed in [42]. After fractal decoding, the embedded watermark diffuses throughout the reconstructed image.

In order to gain high robustness as well as low sensitivity in color image watermarking, the knowledge of human visual perception of color stimuli must be well utilized in designing embedding algorithms. It is well known that the human visual system (HVS) is not perfect sensor of perceiving color visual information. Although the HVS is particularly sensitive to changes in image hue, it is less sensitive to the yellow-blue component. CIE $L a^{*} b^{*}$ is the most complete color model conventionally used to describe all of the colors that are visible to the human eye [10]. The parameter $L$ represents the lightness of the color, whereas $a^{*}, b^{*}$ are chromatic information. And $a^{*}$ is always named magenta-green axis and $b^{*}$ is named yellow-blue axis. Based on the observations, in the method here, we will convert the $r, g$, and $b$ values of the range block means $\left\{\bar{R}_{u}\right\}_{u=1}^{n}$ to $L a^{*} b^{*}$ space before they are inserted watermark $\left\{w_{u}\right\}_{u=1}^{n}$. The transformation from RGB space to the CIE $L a^{*} b^{*}$ space is through the XYZ space. The RGB space is first converted to the $X Y Z$ space through a linear transformation

$$
\left[\begin{array}{l}
X \\
Y \\
Z
\end{array}\right]=\left[\begin{array}{lll}
0.490 & 0.310 & 0.200 \\
0.177 & 0.813 & 0.011 \\
0.000 & 0.010 & 0.990
\end{array}\right]\left[\begin{array}{l}
R \\
G \\
B
\end{array}\right]
$$

and the $X Y Z$ space is converted to the CIE- $L a^{*} b^{*}$ space through a nonlinear transformation

$$
\begin{gathered}
L=166 f\left(\frac{Y}{Y_{0}}\right)-16, \\
a^{*}=500\left[f\left(\frac{X}{X_{0}}\right)-f\left(\frac{Y}{Y_{0}}\right)\right], \\
b^{*}=200\left[f\left(\frac{Y}{Y_{0}}\right)-f\left(\frac{Z}{Z_{0}}\right)\right],
\end{gathered}
$$

where

$$
f(t)= \begin{cases}t^{1 / 3}, & \text { if } t>0.008856 \\ 7.787 x+\frac{16}{116}, & \text { otherwise }\end{cases}
$$




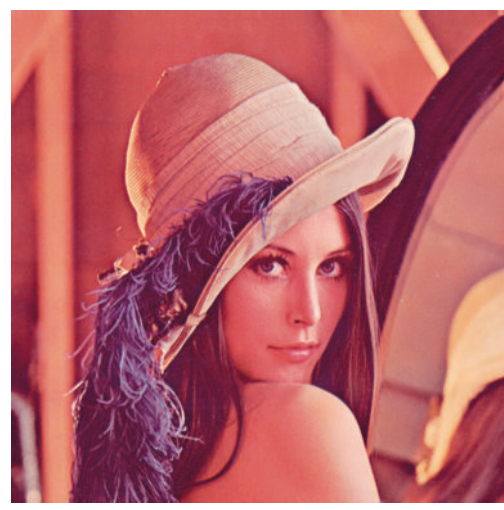

(a) Lena

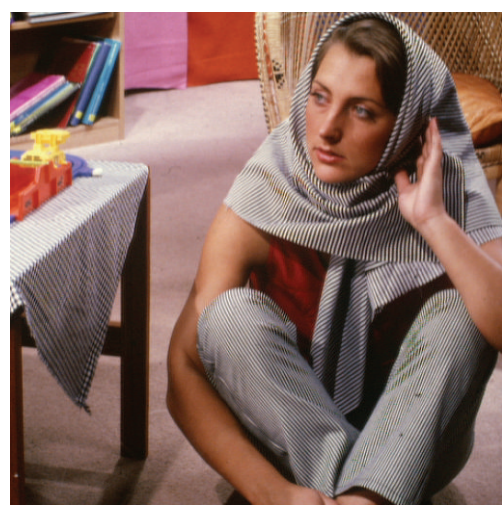

(d) Barbara

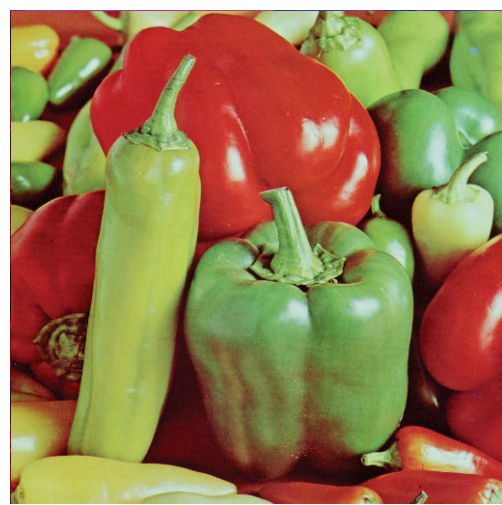

(g) Peppers

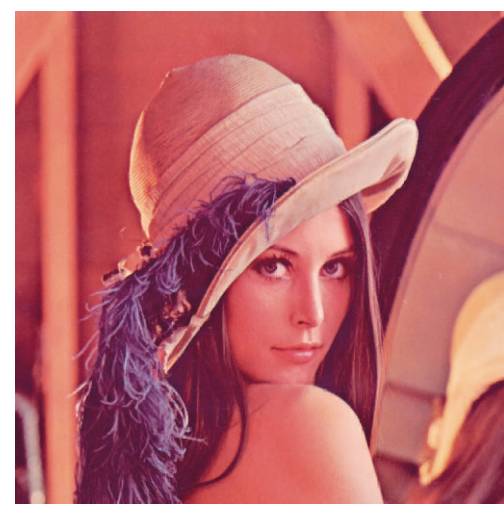

(b) PSNR $34.71 \mathrm{~dB}$

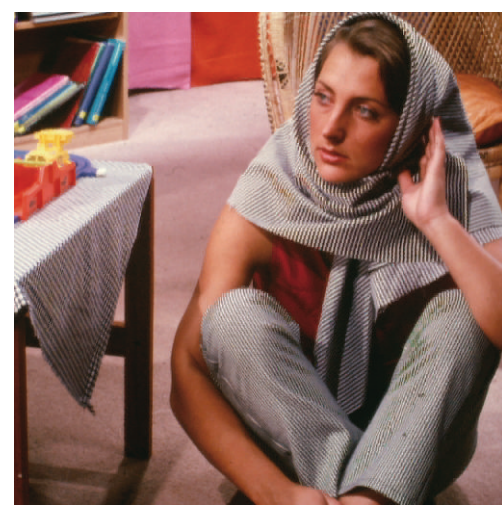

(e) PSNR $30.37 \mathrm{~dB}$

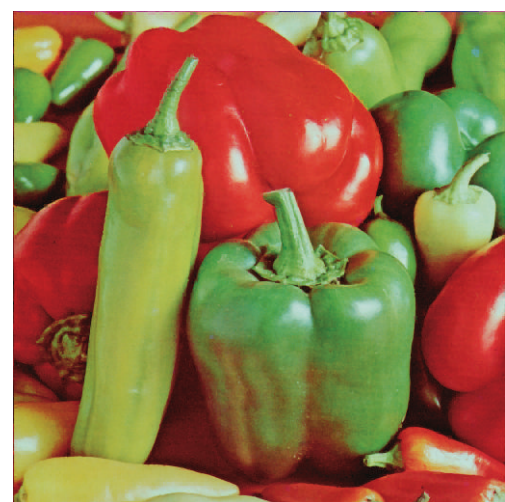

(h) PSNR $32.01 \mathrm{~dB}$

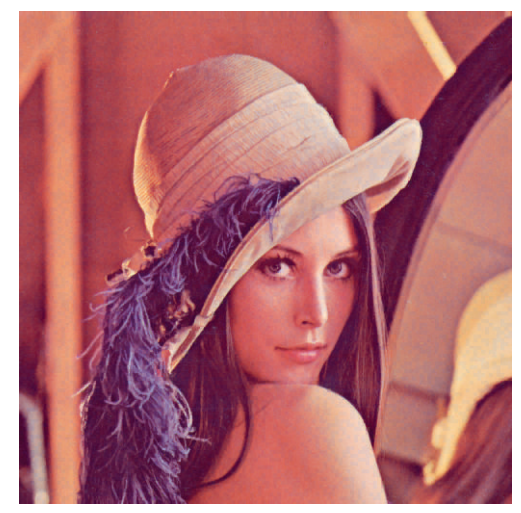

(c) PSNR $32.54 \mathrm{~dB}$

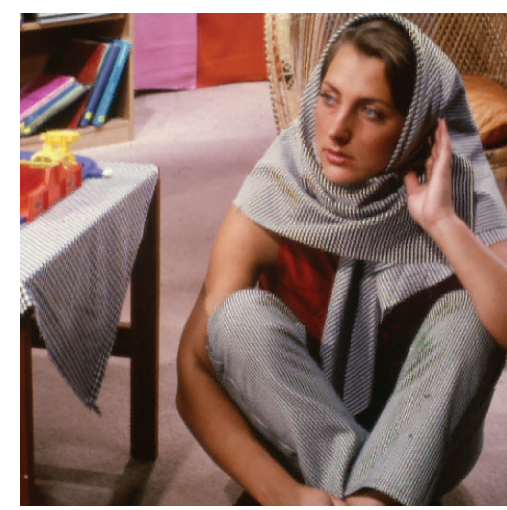

(f) PSNR $28.57 \mathrm{~dB}$

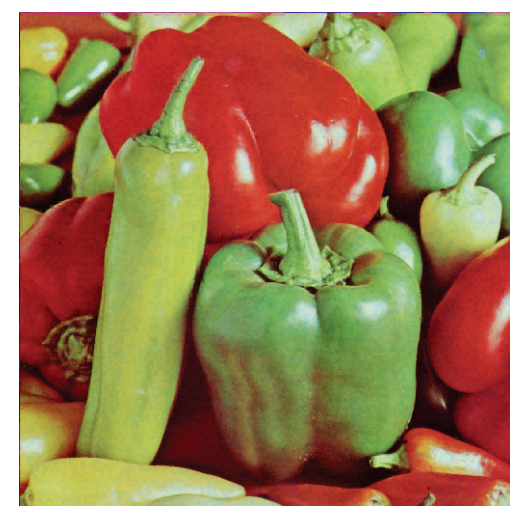

(i) PSNR $30.35 \mathrm{~dB}$

Figure 2: Comparisons of the two fractal color image coding methods: (a), (d) and (g) are original images; (b), (e) and (h) are the fractal representations of our proposed methods; (c), (f) and (i) are the fractal representations of the classic method [2].

and $X_{0}, Y_{0}, Z_{0}$ represent the reference white. Conversely, the reverse transformation is easily expressed by using the inverse of the function $f$ above:

$$
\begin{gathered}
Y=Y_{0} f^{-1}\left(\frac{(L+16)}{116}\right), \\
X=X_{0} f^{-1}\left(\frac{(L+16)}{116}+\frac{a^{*}}{500}\right), \\
Z=Z_{0} f^{-1}\left(\frac{(L+16)}{116}-\frac{b^{*}}{200}\right),
\end{gathered}
$$

where

$$
f^{-1}(t)= \begin{cases}t^{3}, & \text { if } t>0.2069 \\ 0.1284\left(t-\frac{4}{29}\right), & \text { otherwise. }\end{cases}
$$

The color difference $\Delta E$ at each pixel is defined as

$$
\Delta E=\sqrt{(\Delta L)^{2}+\left(\Delta a^{*}\right)^{2}+\left(\Delta b^{*}\right)^{2}} .
$$

Under a specific viewing condition, there exits a certain amount of information that is not perceivable by the human 


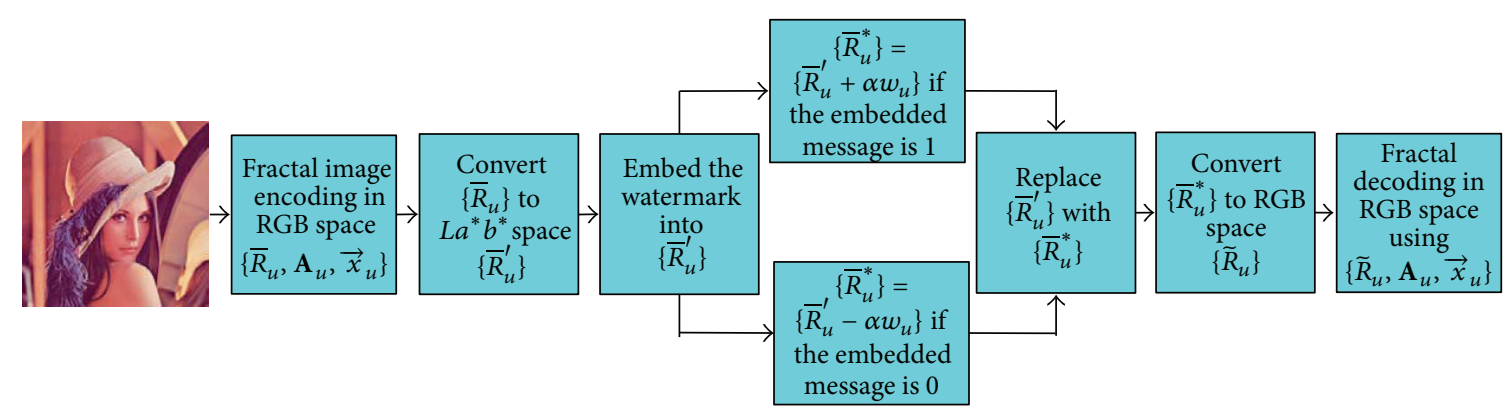

FIGURE 3: Watermark insertion procedure using our fractal color image coding.

eyes, or the so-called perceptual redundancy. Experiments have shown that $\Delta E<1$ is not detectable by the HVS, and $\Delta E<3$ is not apparent [46]. $\Delta E=3$ is called the uniform justnoticeable color difference (UJNCD). Since the sensitivity of the HVS to the yellow-blue component is approximately $1 / 5$ compared to the luminance component [10], we can set $\Delta L=$ $\Delta a^{*}=1 / 5 \Delta b^{*}$. So, if we set $\Delta b^{*}=1.5$, then $\Delta L=\Delta a^{*}=0.3$ and $\Delta E \approx 1.56 \ll 3$. Assume a randomly scrambled watermark to be $\left\{w_{u}\right\}_{u=1}^{n}$ the watermark insertion procedure is illustrated in Figure 3 and includes the following steps.

(1) Fractally encode the original image (using full search scheme) to produce the fractal codes $\left\{\bar{R}_{u}, \mathbf{A}_{u}, \vec{x}_{u}\right\}_{u=1}^{n}$ in $R G B$ space, where $\vec{x}_{u} \doteq\left(x_{u}, y_{u}\right)$ is the position of the optimal domain block.

(2) Convert the range block means $\left\{\bar{R}_{u}\right\}_{u=1}^{n}$ from $R G B$ space to $L a^{*} b^{*}$ space and denote them by $\left\{\bar{R}_{u}^{\prime}\right\}_{u=1}^{n}$.

(3) Embed the permutated watermark $\left\{w_{u}\right\}_{u=1}^{n}$ into $\left\{\bar{R}_{u}^{\prime}\right\}_{u=1}^{n}$.

(i) If the message to be embedded is $w_{u}=1$, replace $\bar{R}_{u}^{\prime}$ with $\bar{R}_{u}^{*} \doteq \bar{R}_{u}^{\prime}+\zeta$, where $\zeta=[0.3,0.3,1.5]^{T}$.

(ii) Otherwise, if $w_{u}=0$, replace $\bar{R}_{u}^{\prime}$ with $\bar{R}_{u}^{*} \doteq \bar{R}_{u}^{\prime}-$ $\zeta$.

(4) Convert $\left\{\bar{R}_{u}^{*}\right\}_{u=1}^{n}$ back to $R G B$ space and denote them by $\left\{\widetilde{R}_{u}^{*}\right\}_{u=1}^{n}$.

(5) Hide the watermark by performing fractal decoding using $\left\{\widetilde{R}_{u}, \mathbf{A}_{u}, \vec{x}_{u}\right\}_{u=1}^{n}$.

To extract the embedded watermark, the original image and watermarked (possibly attacked) image are needed. $\bar{R}_{u}^{\prime}+\zeta$ and $\bar{R}_{u}^{\prime}-\zeta$ are calculated from the original image in $L a^{*} b^{*}$ space, followed by a distance comparison to the $\widetilde{R}_{u}^{\prime}$ from the watermarked image. If the extracted $\widetilde{R}_{u}^{\prime}$ is closer to $\bar{R}_{u}^{\prime}+\zeta$, we regard " 1 " as embedded; otherwise, "0" was inserted. The flow diagram is illustrated in Figure 4. The watermark extraction is very quick since it does not need fractal encoding and decoding.

\section{Experimental Results}

In order to verify that the proposed algorithm described before indeed increases the robustness of the watermarked images against attacks, a series of experiments has been conducted by using the attacks to the $512 \times 512$ color Lena, Peppers, and Barbara images, as shown in Figures 2(a), 2(d), and $2(\mathrm{~g})$. The size of the color range block is $4 \times 4$, so the original image can be partitioned into $128 \times 128$ (i.e., 16384) color range blocks. For convenience, the size of binary logo image to be embedded is $128 \times 128$, and the logo image as shown in Figure 5(a), a lovely panda, is encrypted by using the block shifting method [47]. That is, we first divide the logo image into $32 \times 32$ small blocks, each with $4 \times 4$ pixels. Successively, the positions of the small blocks are randomly shifted by a random matrix, such as

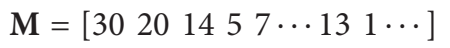

which is generated by randomly scrambling the integers $1,2, \ldots, 32$. As an example used here, the resulting image is shown in Figure 5(b). If people want to recover the original information, the same random matrix should be used,; otherwise people could not obtain the correct information.

The attacked image quality is measured by peak signal-tonoise ratio (PSNR) defined as

$$
\mathrm{PSNR}=10 \log _{10} \frac{255^{2}}{\mathrm{MSE}},
$$

where

$$
\operatorname{MSE}=\frac{1}{3 f_{H} \times f_{W}} \sum_{i=1}^{f_{H}} \sum_{j=1}^{f_{W}}\|f(i, j)-\tilde{f}(i, j)\|_{2}^{2} .
$$

Here, $f_{H}$ and $f_{W}$ are the height and width of the image, respectively. $f(i, j)$ and $\widetilde{f}(i, j)$ are the values located at coordinates $(i, j)$ of the original image and the attacked image, respectively.

When extracting the watermark, the normalized correlation coefficient (NC) is calculated using the original watermark and the extracted watermark to judge the existence of the watermark and to measure the correctness of the extracted watermark. It is defined as

$$
\mathrm{NC}=\frac{1}{w_{h} \times w_{w}} \sum_{i=1}^{w_{h}} \sum_{j=1}^{w_{w}} w(i, j) \widetilde{w}(i, j),
$$




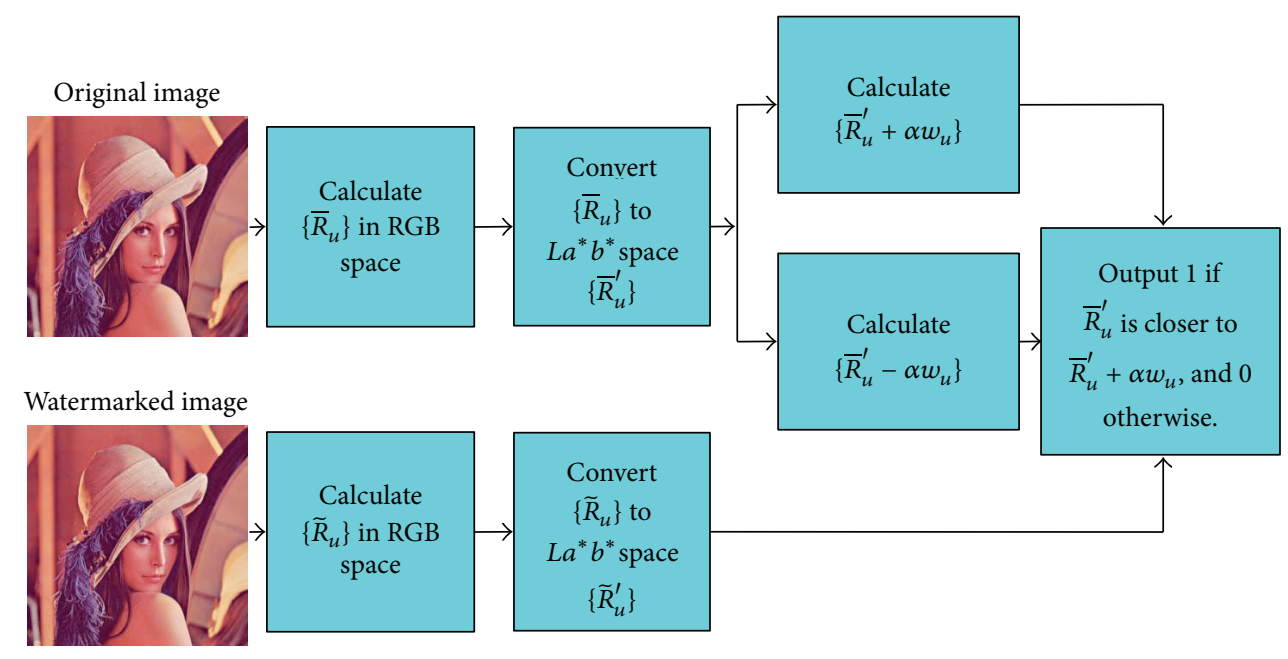

FIGURE 4: Watermark extraction procedure.

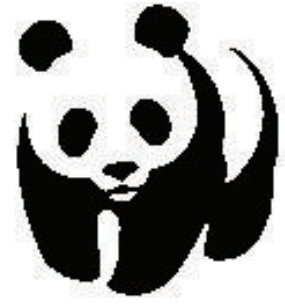

(a)

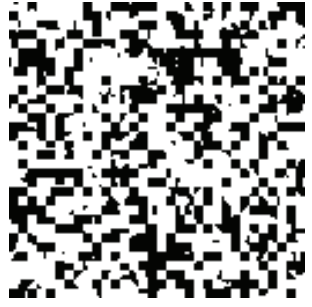

(b)
FIGURE 5: (a) Binary watermark logo. (b) Randomly scrambled logo.

where $w_{h}$ and $w_{w}$ are the height and width of the watermark, respectively. $w(i, j)$ and $\widetilde{w}(i, j)$ are the watermark bits located at $(i, j)$ of the original watermark and the extracted watermark. And $w(i, j)$ is set to 1 if it is watermark bit 1 ; otherwise, it is set to -1 . Likewise for $\widetilde{w}(i, j)$. The PSNRs of the three watermarked images are $33.30 \mathrm{~dB}, 29.05 \mathrm{~dB}$, and $30.59 \mathrm{~dB}$, respectively. For brevity, only the Lena image is shown. Figure 6(a) shows that in the unattacked watermarked image, no visual difference exists at all compared with Figure 2(b). The extracted logo image shown in Figure 6(b) with $\mathrm{NC}=1.0$ implies that the extracted watermark is exactly the same as the original watermark.

Now, various classic image-processing operations, including JPEG compression, median filtering, mean filtering, sharping, blurring, noising, cropping, and scaling, are simulated to investigate the robustness of the proposed watermarking method. JPEG is one of the most frequently used image compression formats. The JPEG quality factor is a number between 0 and 100 . When decreasing the quality factor, the image compression ratio increases, whereas the quality of the resulting image is significantly reduced. Table 1 lists the results of applying various JPEG quality factors to the test images. The proposed method can detect the existence of a watermark even for quality factor equal to 15 . And the values of NC still exceed 0.49 for the three images. For other nongeometric attacks, such as median filters, mean filters,

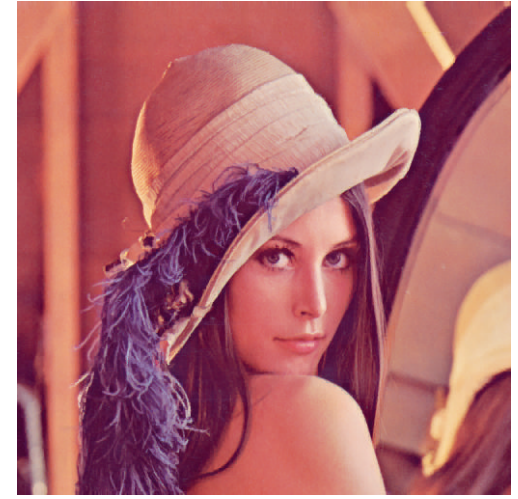

(a)

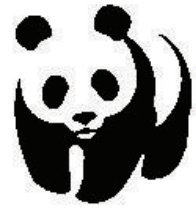

(b)
FIgURE 6: (a) Watermarked Lena image, PSNR $=33.30 \mathrm{~dB}$. (b) Extracted watermark logo, $\mathrm{NC}=1.0$.

sharpening, and blurring, the resulting images are blurred or sharpened at the edge and so forth. The extracted watermarks are illustrated in Table 2. Here, both the median and mean filters are applied with masks of size $3 \times 3,5 \times 5$ and $7 \times 7$, respectively. As we can see, the proposed method is very robust against these non-geometric attacks and most of the extracted logo images are only slightly contaminated.

The results of noising and some common geometric attacks, for example, rotation, scaling and, cropping, are illustrated in Table 3. The Gaussian noise variation is varied from 5 to 15 and the step size is 5 . As shown, the proposed method can effectively resist the attacks and all of the extracted watermarks could be clearly recognized. The attack of scaling is performed by downsampling a watermarked color image by factor 2 in both row and column directions. Since the image is shrunk from size $512 \times 512$ to $256 \times 256$, all range blocks are then contracted from $4 \times 4$ to $2 \times 2$ and $\left\{\widetilde{R}_{u}\right\}_{u=1}^{n}$ should be calculated in a $2 \times 2$ block. For the clopping attack in our test, three types, namely, Type I, Type II, and Type III, are considered. In Type I, the left-top of an image of quarter size 
TABLE 1: Extracted watermarks (EW) and NCs after attacked by JPEG compression with the quality factors (QF) $15,25,35,45,55,65,75$, and 85 on Lena, Barbara, and Peppers.

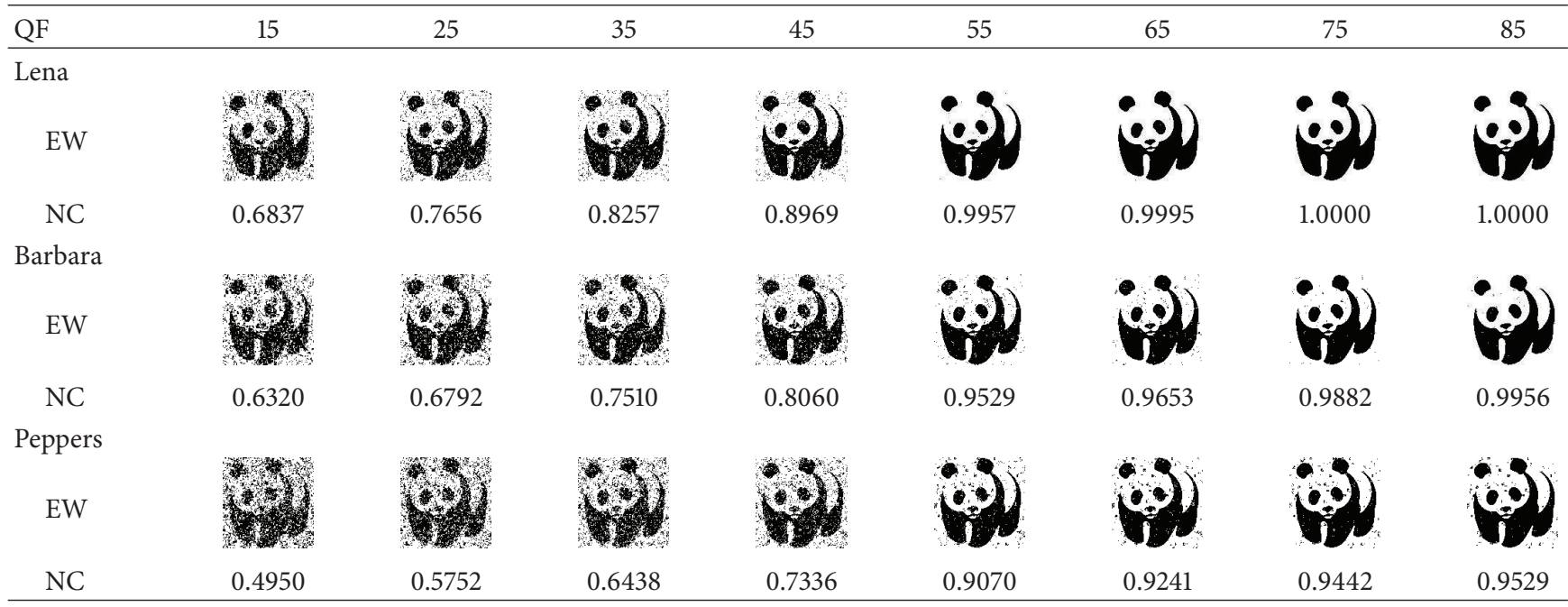

TABLE 2: Extracted watermarks (EW) and NCs after attacked by median filter $(3 \times 3,5 \times 5,7 \times 7)$, mean filter $(3 \times 3,5 \times 5,7 \times 7)$, sharpening, and blurring of the three images: Lena, Barbara, and Peppers.

\begin{tabular}{|c|c|c|c|c|c|c|c|c|}
\hline & & edian fi & & & Tean filte & & Shornening & Plurring \\
\hline & $3 \times 3$ & $5 \times 5$ & $7 \times 7$ & $3 \times 3$ & $5 \times 5$ & $7 \times 7$ & олатретाт & Diunin \\
\hline Lena & & & & & & & & \\
\hline EW & & & & & & & & \\
\hline NC & 0.9937 & 0.9421 & 0.8593 & 0.9764 & 0.8832 & 0.7648 & 0.9872 & 0.9847 \\
\hline Barbara & & & & & & & & \\
\hline EW & & & & & & & & \\
\hline $\mathrm{NC}$ & 0.9966 & 0.9502 & 0.8641 & 0.9796 & 0.8837 & 0.7561 & 0.9957 & 0.9900 \\
\hline Peppers & & & & & & & & \\
\hline EW & & & & & & (e) & & \\
\hline NC & 0.9780 & 0.9288 & 0.8456 & 0.9437 & 0.8392 & 0.7111 & 0.9675 & 0.9629 \\
\hline
\end{tabular}

is cut away (i.e., 75\% is remained), as shown in Figure 7(a); in Type II, the positions of the left-top and the right-bottom of the remained subimage are $(31,471)$ and $(81,461)$, respectively. In other words, the remaining region is about $64 \%$, as shown in Figure 7(b); in Type III, two rectangular regions are removed and the remaining part is about $79 \%$, as shown in Figure $7(\mathrm{c})$. In practical use, the position of the clipped block is usually needed to be located, especially for the case of Type II. Similar to the method in [42], it can be easily achieved by moving the clipped block point by point from the top-left of watermarked image and calculating the correlation coefficient. Clearly, the position of the clipped block should be located at the position where the correlation coefficient is maximum (theoretically, it equals 1 if without any other attacks). Then, in order to facilitate obtaining the subwatermark of the clipped block correctly, the vacant region is filled up with 0 (black). Hence, the size of the patched color image is still $512 \times 512$. Finally, the watermark extraction procedure is performed and the NC value of the watermark is calculated, as described in Section 4. The results are also listed in Table 3. It is worth noticing that the NC value is closely related with the size of clipped block: smaller size of the clipped block generally means smaller $\mathrm{NC}$ value. So, a more reasonable method for detecting the existence of a watermark in the clipped block is only computing the NC value of the subwatermark of the clipped region (it is almost 1 in theory).

We next compare the proposed method to three traditional methods: Tsui et al. [15], Kutter et al. [12] and Cox et al's [5] methods using the Lena image. The results are shown in Table 4. In the comparisons, all the methods still use the panda as the test binary logo image, but the size becomes $64 \times$ 64 , which can be obtained by downsampling the original 


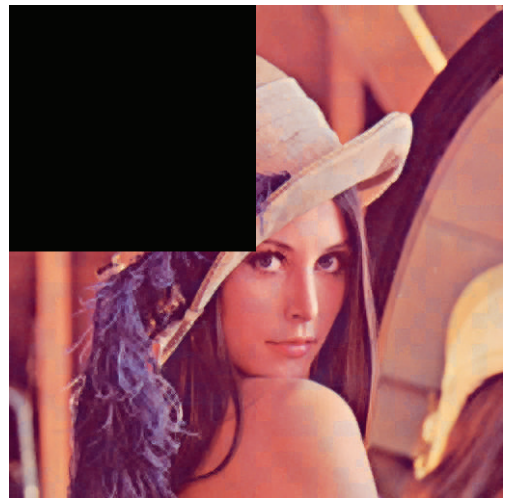

(a) Type I

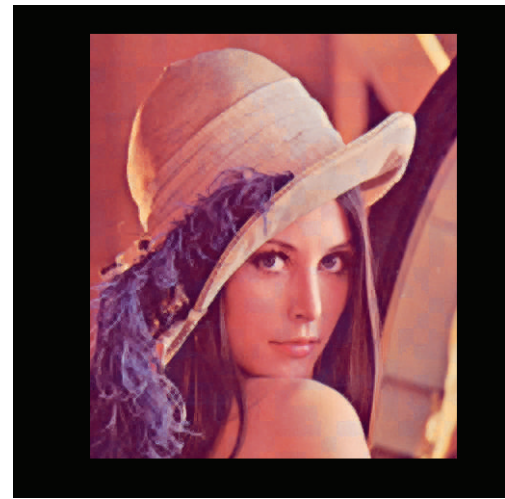

(b) Type II

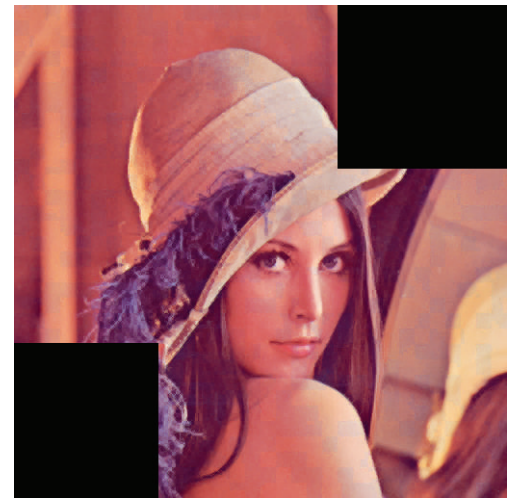

(c) Type III

FIGURE 7: Attacked watermarked Lena image by various croppings.

TABLE 3: Extracted watermarks (EW) and NCs after attacked by Gaussian noise added from $\sigma=5$ to 10 , and to 15 , scaling with factor 2, and various cropping to the original size of the three images.

\begin{tabular}{|c|c|c|c|c|c|c|c|}
\hline & \multicolumn{3}{|c|}{ Gaussian noise } & \multicolumn{3}{|c|}{ Cropping (Figure 7) } & \multirow{2}{*}{$\begin{array}{l}\text { Scaling } \\
\text { (factor 2) }\end{array}$} \\
\hline & $\sigma=5$ & $\sigma=10$ & $\sigma=15$ & I & II & III & \\
\hline \multicolumn{8}{|l|}{ Lena } \\
\hline \multicolumn{8}{|l|}{ EW } \\
\hline $\mathrm{NC}$ & 0.9803 & 0.8225 & 0.6276 & 0.6997 & 0.6075 & 0.7957 & 0.9633 \\
\hline \multicolumn{8}{|l|}{ Barbara } \\
\hline \multicolumn{8}{|c|}{$6(x)$} \\
\hline $\mathrm{NC}$ & 0.9148 & 0.7429 & 0.5657 & 0.7086 & 0.5800 & 0.7463 & 0.9767 \\
\hline \multicolumn{8}{|c|}{ Peppers } \\
\hline FW & & $(69)$ & $W^{2}\left(x^{2}\right)$ & 48 & ats & $0 \%$ & \\
\hline & & & (6) & & 4 和 & & \\
\hline $\mathrm{NC}$ & 0.9010 & 0.7024 & 0.5280 & 0.6959 & 0.5665 & 0.7396 & 0.9480 \\
\hline
\end{tabular}

TABLE 4: Comparing the proposed method with Tsui at al. [15], Kutter et al. [12] and Cox at al. [5] methods.

\begin{tabular}{|c|c|c|c|c|}
\hline Attacks & Tsui et al. [15] & Kutter et al. [12] & Cox et al. [5] & Proposed \\
\hline Attack free & 1.0 & 0.9476 & 0.9957 & 1.0 \\
\hline Median filter $(3 \times 3)$ & 0.9877 & 0.8750 & 0.9146 & 0.9936 \\
\hline \multicolumn{5}{|l|}{ JPEG } \\
\hline 10 & 0.4237 & N/A & 0.6889 & 0.7001 \\
\hline 50 & 0.9302 & 0.3504 & 0.9396 & 0.9401 \\
\hline Gaussian noise $(\sigma=20)$ & 0.5038 & 0.4788 & 0.4516 & 0.5124 \\
\hline Scaling (factor 2) & 0.8743 & 0.8500 & 0.8460 & 0.9633 \\
\hline Sharpening & 0.9781 & 0.8893 & 0.9405 & 0.9872 \\
\hline Bluring & 0.9650 & 0.8956 & 0.9463 & 0.9847 \\
\hline Cropping & 0.6981 & 0.6330 & 0.5954 & 0.6990 \\
\hline
\end{tabular}


$128 \times 128$ logo image. In our proposed method, the logo is needed to be expanded to $128 \times 128$ size by filling up with 0 on its three neighboring regions: left, bottom, and leftbottom, before it is embedded to the Lena image during the embedding procedure. Correspondingly, at the end of the extracting process, the logo is obtained by clipping the extracted logo image on the left-top with the size of $64 \times 64$. As shown in Table 4 , the proposed method generally outperforms the other three methods for various attacks.

\section{Conclusion}

In this work, we have developed a fractal color image watermarking method and assessed its performance. As we know, the main objectives of watermarking are to achieve a good robustness against attacks and retain high image quality as well. In the proposed method, we consider a pixel as a 3-D vector and an $R G B$ version of orthogonalization fractal coding is performed in $R G B$ space. In order to obtain higher image quality, instead of using three independent linear functions in classic fractal color coding, we utilize a general form of fractal affine transform by using the range block mean vector as the luminance offset and a $3 \times 3$ matrix as contrast scaling. In terms of the image quality measured by PSNR, the proposed fractal color coding significantly outperforms the traditional one. It is worth noting that, since the compression ratio is not the main point for watermarking, we do not discuss the quantization of the fractal parameters. On the other hand, we also show that the orthogonalization fractal color decoding is a mean vector-invariant iteration. Hence, the range block mean vector is a good place for embedding the watermark. For the sake of consistency with the human visual system and ensuring the watermark invisibility as well as possible, we further implement the hiding procedure in the CIE $L a^{*} b^{*}$ space and incorporate a JND scheme. Our experiments show that the proposed watermarking has good robustness against various distortions such as JPEG compression, median filtering, and geometric distortion such as scaling and cropping, as well as with an imperceptible change in image quality.

\section{Conflict of Interests}

The authors declare that there is no conflict of interests regarding the publication of this paper.

\section{Acknowledgments}

The authors would like to thank the anonymous referees for their helpful comments and suggestions that led to a significant improvement of the paper. This work was supported by the National Natural Science Foundation of China (nos. 61003178, 61070087, 61373087, 11201312, 61272252, and 11071150), and by the Municipal Science and Technology Plan of Shenzhen in China (JC201105170615A, JC201005280508A).

\section{References}

[1] V. M. Potdar, S. Han, and E. Chang, "A survey of digital image watermarking techniques," in Proceedings of the 3rd IEEE International Conference on Industrial Informatics (INDIN '05), pp. 709-716, Perth, Australia, August 2005.

[2] Y. Zhang and L.-M. Po, "Fractal color image compression using vector distortion measure," in Proceedings of the IEEE International Conference on Image Processing (ICIP '95), vol. 3, pp. 276279, Washington, DC, USA, October 1995.

[3] B. Chen and G. W. Wornell, "Quantization index modulation methods for digital watermarking and information embedding of multimedia," Journal of VLSI Signal Processing Systems for Signal, Image, and Video Technology, vol. 27, no. 1-2, pp. 7-33, 2001.

[4] R. Ye and S. Wu, "Application of chaotic ergodicity in image encryption and watermarking," in Proceedings of the 2nd International Conference on Mechanic Automation and Control Engineering (MACE '11), pp. 7196-7199, Inner Mongolia, China, July 2011.

[5] I. J. Cox, J. Kilian, F. T. Leighton, and T. Shamoon, "Secure spread spectrum watermarking for multimedia," IEEE Transactions on Image Processing, vol. 6, no. 12, pp. 1673-1687, 1997.

[6] N. Ahmidi and R. Safabakhsh, "A novel DCT-based approach for secure color image watermarking," in Proceedings of the International Conference on Information Technology: Coding Computing (ITCC '04), vol. 2, pp. 709-713, Las Vegas, Nev, USA, April 2004.

[7] V. Solachidis and I. Pitas, "Circularly symmetric watermark embedding in 2-D DFT domain," IEEE Transactions on Image Processing, vol. 10, no. 11, pp. 1741-1753, 2001.

[8] W.-H. Lin, Y.-R. Wang, S.-J. Horng, T.-W. Kao, and Y. Pan, "A blind watermarking method using maximum wavelet coefficient quantization," Expert Systems with Applications, vol. 36, no. 9, pp. 11509-11516, 2009.

[9] H. A. Al-Otum and A. O. Al-Taba'a, "Adaptive color image watermarking based on a modified improved pixel-wise masking technique," Computers and Electrical Engineering, vol. 35, no. 5, pp. 673-695, 2009.

[10] B. A. Wandell, Foundations of Vision, Sinauer Associates, Sunderland, Mass, USA, 1995.

[11] C. P. Li, Y. J. Wu, and R. S. Ye, Eds., Recent Advances in Applied Nonlinear Dynamics with Numerical Analysis, World Scientific Publishing, Singapore, 2013.

[12] M. Kutter, F. Jordan, and F. Bossen, "Digital watermarking of color images using amplitude modulation," Journal of Electronic Imaging, vol. 7, no. 2, pp. 326-332, 1998.

[13] M. Barni, F. Bartolini, and A. Piva, "Multichannel watermarking of color images," IEEE Transactions on Circuits and Systems for Video Technology, vol. 12, no. 3, pp. 142-156, 2002.

[14] P. Tsai, Y.-C. Hu, and C.-C. Chang, "A color image watermarking scheme based on color quantization," Signal Processing, vol. 84, no. 1, pp. 95-106, 2004.

[15] T. K. Tsui, X.-P. Zhang, and D. Androutsos, "Color image watermarking using the spatio-chromatic fourier transform," in Proceedings of the IEEE International Conference on Acoustics, Speech and Signal Processing (ICASSP '06), pp. 305-308, Toulouse, France, May 2006.

[16] C.-T. Kuo and S.-C. Cheng, "Fusion of color edge detection and color quantization for color image watermarking using principal axes analysis," Pattern Recognition, vol. 40, no. 12, pp. 36913704, 2007. 
[17] C.-H. Chou and K.-C. Liu, "A perceptually tuned watermarking scheme for color images," IEEE Transactions on Image Processing, vol. 19, no. 11, pp. 2966-2982, 2010.

[18] E. Vahedi, R. A. Zoroofi, and M. Shiva, “Toward a new waveletbased watermarking approach for color images using bioinspired optimization principles," Digital Signal Processing, vol. 22, no. 1, pp. 153-162, 2012.

[19] M. F. Barnsley and S. Demko, "Iterated function systems and the global construction of fractals," Proceedings of the Royal Society A, vol. 399, no. 1817, pp. 243-275, 1985.

[20] A. E. Jacquin, "Image coding based on a fractal theory of iterated contractive image transformations," IEEE Transactions of Image Processing, vol. 1, no. 1, pp. 18-30, 1992.

[21] Y. Fisher, Fractal Image Compression: Theory and Applications, Springer, Berlin, Germany, 1995.

[22] C.-M. Lai, K.-M. Lam, and W.-C. Siu, "A fast fractal image coding based on kick-out and zero contrast conditions," IEEE Transactions on Image Processing, vol. 12, no. 11, pp. 1398-1403, 2003.

[23] X.-Y. Wang, F.-P. Li, and Z.-F. Chen, "An improved fractal image coding method," Fractals, vol. 17, no. 4, pp. 451-457, 2009.

[24] X. Wang, F. Li, and S. Wang, "Fractal image compression based on spatial correlation and hybrid genetic algorithm," Journal of Visual Communication and Image Representation, vol. 20, no. 8, pp. 505-510, 2009.

[25] C.-C. Tseng, J.-G. Hsieh, and J.-H. Jeng, "Fractal image compression using visual-based particle swarm optimization," Image and Vision Computing, vol. 26, no. 8, pp. 1154-1162, 2008.

[26] Y. Zhang and X. Wang, "Fractal compression coding based on wavelet transform with diamond search," Nonlinear Analysis: Real World Applications, vol. 13, no. 1, pp. 106-112, 2012.

[27] C. S. Tong and M. Pi, "Fast fractal image encoding based on adaptive search," IEEE Transactions on Image Processing, vol. 10, no. 9, pp. 1269-1277, 2001.

[28] X.-Y. Wang and L.-X. Zou, "Fractal image compression based on matching error threshold," Fractals, vol. 17, no. 1, pp. 109-115, 2009.

[29] H.-N. Chen, K.-L. Chung, and J.-E. Hung, "Novel fractal image encoding algorithm using normalized one-norm and kick-out condition," Image and Vision Computing, vol. 28, no. 3, pp. 518$525,2010$.

[30] X. Y. Wang, Y. X. Wang, and J. J. Yun, "An improved no-search fractal image coding method based on a fitting plane," Image and Vision Computing, vol. 28, no. 8, pp. 1303-1308, 2010.

[31] X. Wang, D. Zhang, and X. Guo, "Novel hybrid fractal image encoding algorithm using standard deviation and DCT coefficients," Nonlinear Dynamics, vol. 73, no. 1-2, pp. 347-355, 2013.

[32] X. Y. Wang, X. Guo, and D. D. Zhang, "An effective fractal image compression algorithm based on plane fitting," Chinese Physics B, vol. 21, no. 9, Article ID 090507, 2012.

[33] X. Y. Wang, Y. X. Wang, and J. J. Yun, "An improved no-search fractal image coding method based on a modified gray-level transform," Computers \& Graphics, vol. 32, no. 4, pp. 445-450, 2008.

[34] M. Ghazel, G. H. Freeman, and E. R. Vrscay, "Fractal image denoising," IEEE Transactions on Image Processing, vol. 12, no. 12, pp. 1560-1578, 2003.

[35] J. Lu, Z. Ye, Y. Zou, and R. Ye, "An enhanced fractal image denoising algorithm," Chaos, Solitons \& Fractals, vol. 38, no. 4, pp. 1054-1064, 2008.
[36] J.-H. Jeng, C.-C. Tseng, and J.-G. Hsieh, "Study on Huber fractal image compression," IEEE Transactions on Image Processing, vol. 18, no. 5, pp. 995-1003, 2009.

[37] J. Lu, Z. Ye, and Y. Zou, "Huber fractal image coding based on a fitting plane," IEEE Transactions on Image Processing, vol. 22, no. 1, pp. 134-145, 2013.

[38] M. Pi, M. K. Mandal, and A. Basu, "Image retrieval based on histogram of fractal parameters," IEEE Transactions on Multimedia, vol. 7, no. 4, pp. 597-605, 2005.

[39] P. Davern and M. Scott, "Fractal based image steganography," in Information Hiding, vol. 1174 of Lecture Notes in Ccomputer Science, pp. 279-294, Springer, Berlin, Germany, 1996.

[40] M. Čandik, D. Levický, and Z. Klenovičová, "Fractal image coding with digital watermarks," Radioengieering, vol. 9, no. 4, pp. 22-26, 2000.

[41] S. Kiani and M. E. Moghaddam, "Fractal based digital image watermarking using fuzzy C-mean clustering," in Proceedings of the International Conference on Information Management and Engineering (ICIME '09), pp. 638-642, Kuala Lumpur, Malaysia, April 2009.

[42] M. H. Pi, C. H. Li, and H. Li, "A novel fractal image watermarking," IEEE Transactions on Multimedia, vol. 8, no. 3, pp. 488498, 2006.

[43] S. Shahraeini and M. Yaghoobi, "A robust digital image watermarking approach against JPEG compression attack on bybrid fractal-wavelet," in Proceedings of the International Conference on Computer Communication and Management (ICCCM '11), vol. 5, pp. 616-622, Wuhan, China, 2011.

[44] C.-H. Chou and Y.-C. Li, "A perceptually tuned subband image coder based on the measure of just-noticeable-distortion profile," IEEE Transactions on Circuits and Systems for Video Technology, vol. 5, no. 6, pp. 467-476, 1995.

[45] J. Z. Liu, "Some estimates of correlation coefficients for systems of random vectors," Applied Mathematics, vol. 14, no. 1, pp. 4348, 1999.

[46] C. H. Chou and K. C. Liu, "An oblivious and robust watermarking scheme using perceptual model," in Proceedings of the 4th EURASIP Conference on Video/Image Processing and Multimedia Communications (VIPMC '03), pp. 713-720, Zagreb, Croatia, 2003.

[47] B. Hennelly and J. T. Sheridan, "Optical image encryption by random shifting in fractional Fourier domains," Optics Letters, vol. 28 , no. 4, pp. 269-271, 2003. 


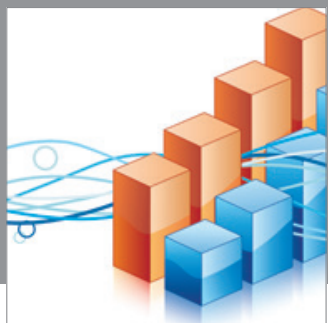

Advances in

Operations Research

mansans

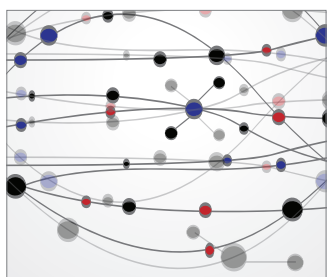

The Scientific World Journal
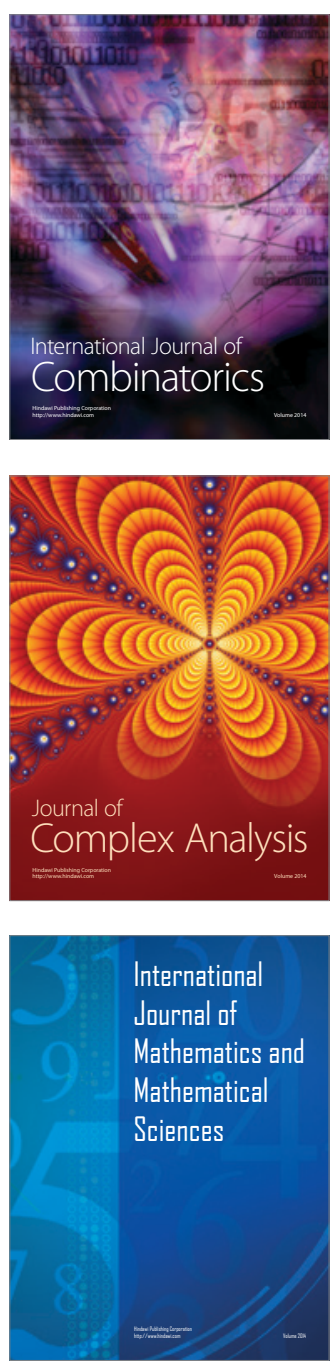
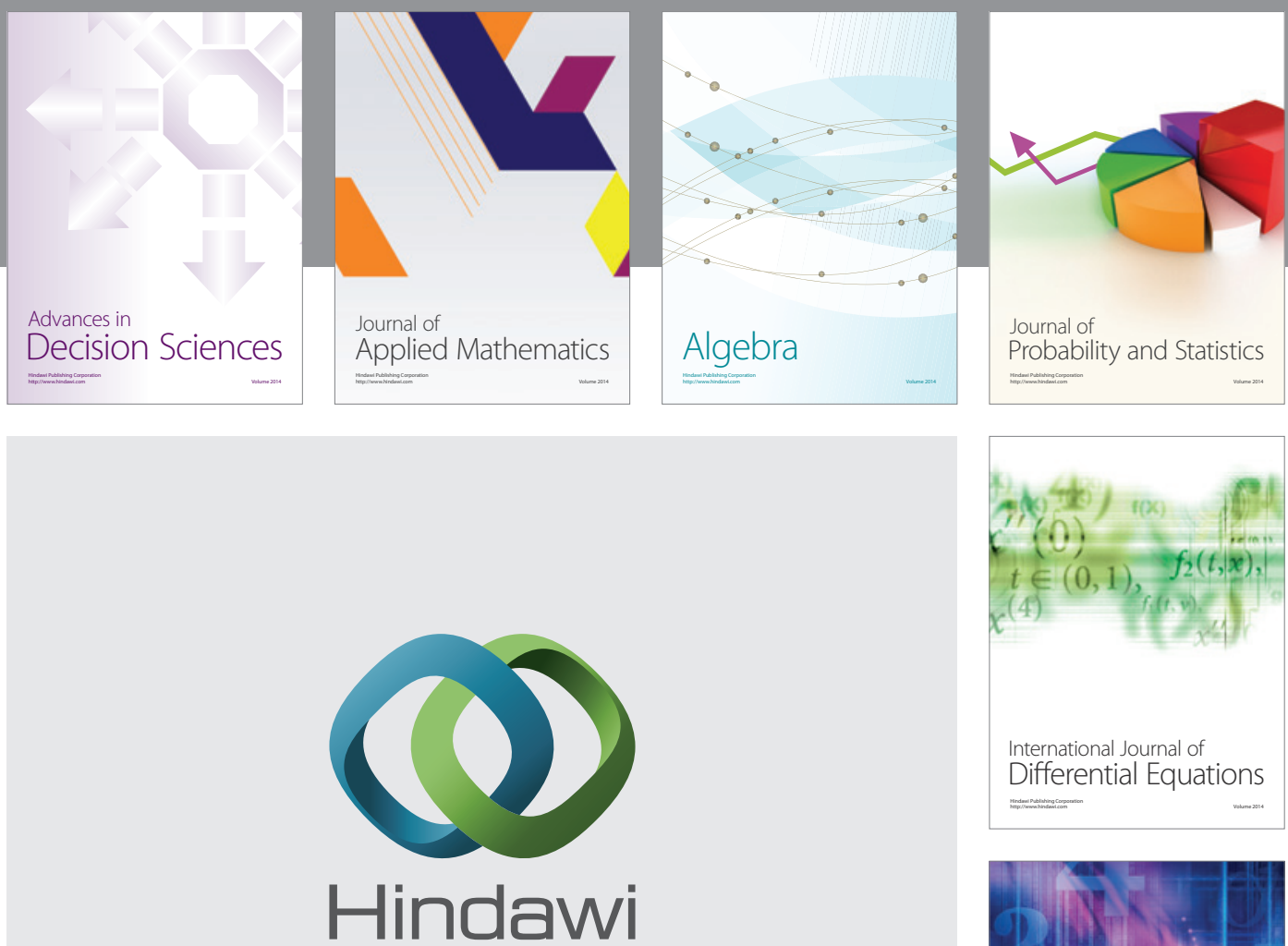

Submit your manuscripts at http://www.hindawi.com
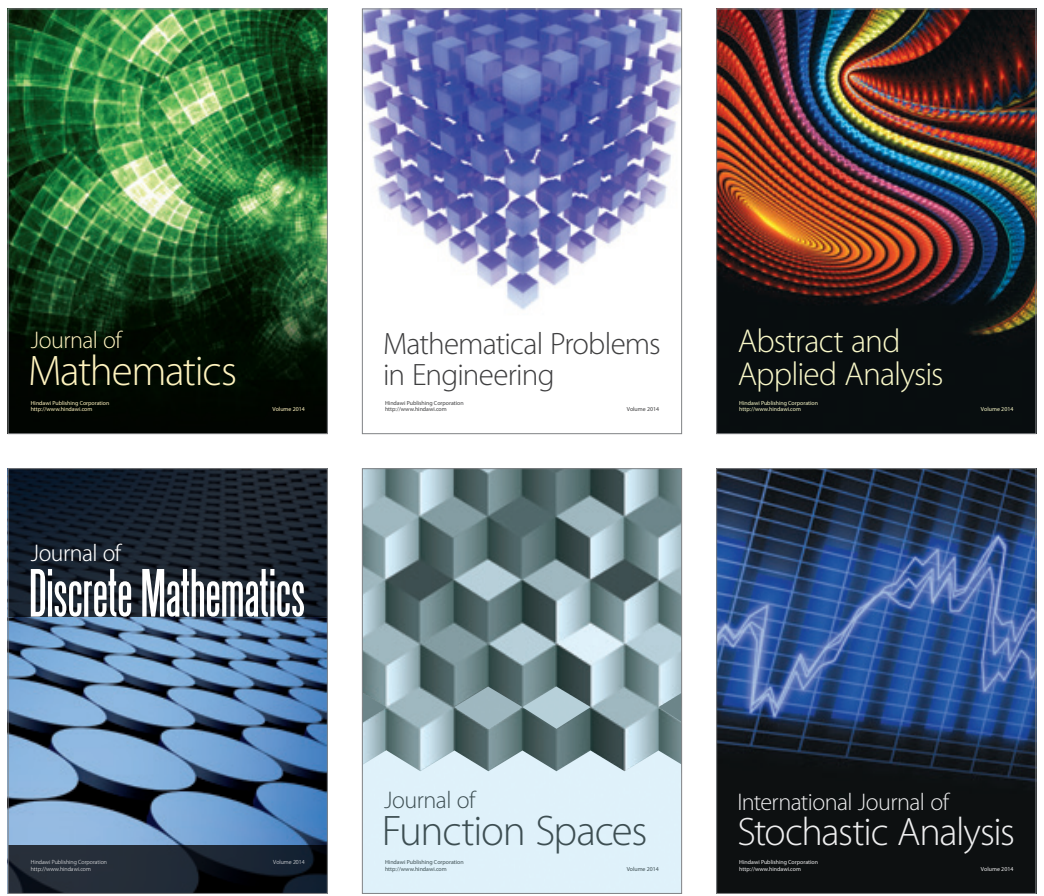

Journal of

Function Spaces

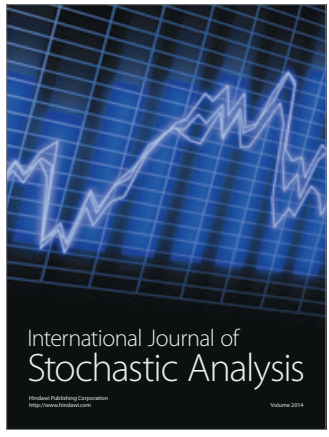

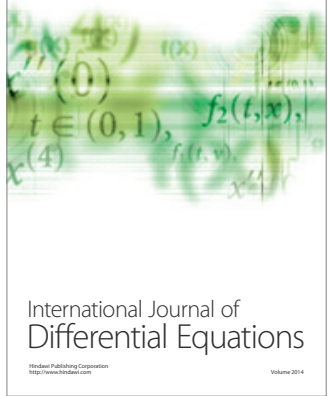
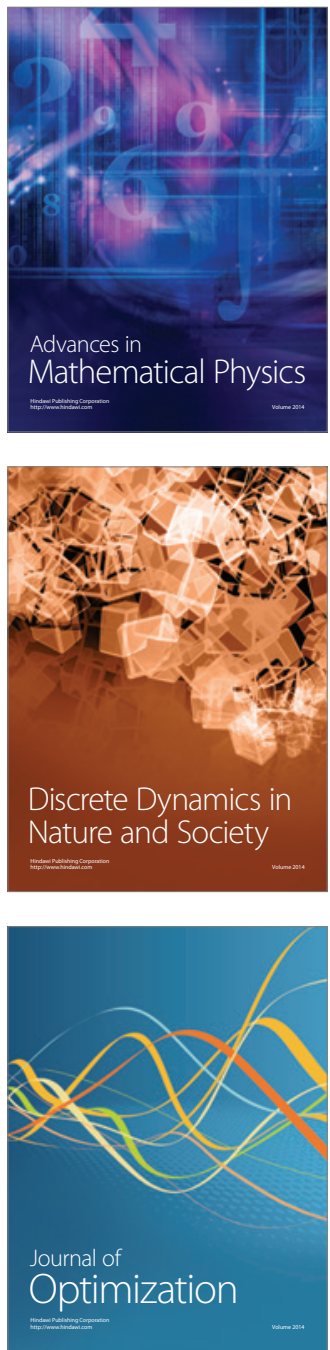